Perspektiven auf den ökonomischen Mainstream

\title{
Die Care-Seite des modernen Kapitalismus
}

\author{
Warum liefert der ökonomische Mainstream \\ kaum Deutungen und Lösungsvorschläge \\ für die soziale Krise? Ansätze der feministischen \\ Ökonomik bieten Erklärungen und einen not- \\ wendigen Perspektivenwechsel, der die mensch- \\ lichen Lebensinteressen ins Zentrum politischen \\ und ökonomischen Handelns rückt. \\ Von Lisa Weinhold
}

W ir leben in Zeiten einer Vielfachkrise, die sich in ökonomischen, ökologischen und politischen Varianten zeigt, die sich wechselseitig bedingen und intensivieren (Demirović et al. 2011). Allerdings wird einer Dimension kaum Beachtung in Politik und Wissenschaft geschenkt: Die Krise der sozialen Reproduktion (Bauhardt 2012; Fraser 2016). Unter sozialer Reproduktion werden einerseits institutionelle Angebote für das Gemeinwohl der Gesellschaft verstanden. Andererseits gehört die Sorge um die eigene Person und andere sowie alle haushaltsnahen Tätigkeiten dazu.

Die Krise der sozialen Reproduktion äußert sich mit Blick auf Deutschland wie folgt: Seit der Integration von Frauen [1] in den Arbeitsmarkt Ende der 1970er aufgrund der Wirtschaftskrise(n) sind insbesondere die finanziellen und zeitlichen Anforderungen der familiären Reproduktionsarbeit gestiegen. Mütter, die immer noch mehr Sorgearbeit übernehmen als Väter (Hobler et al. 2017), müssen neben der Erwerbstätigkeit beispielsweise ihre Kinder zu Sportaktivitäten bringen und sich um Angehörige kümmern.

\section{Feministische Ökonomik: Mehr als eine ökonomische Theorieschule}

Auch die Sorge um die eigene Person verlangt im allgemeinen Optimierungsdruck unserer Gesellschaft ein „immer mehr, immer besser“. Dies überfordert und benachteiligt sowohl die Sorgeleistenden als auch die Sorgeempfangenden (Winker 2013). Wo Sorgearbeit entlohnt wird, steht sie unter einem starken Kostendruck. Aktueller Pflegenotstand oder Kindertagesstätten-Streiks sind nur sichtbarer Ausdruck dessen. Ferner wird das Soziale immer stärker durch marktwirtschaftliche Prozesse geprägt. So müssen vermehrt eigentlich öffentliche Dienstleistungen, wie medizinische Versorgung oder Kinderbetreuung, privat finanziert werden. Die Folgen sind Ver- drängung aus Lebensräumen, Unsicherheit, Verstärkung von sozialen Spaltungen und Diskriminierung. Frauen betrifft dies in der Regel am stärksten. Die (Wirtschafts-)Wissenschaften und Politik bieten allerdings kaum Deutungs- und Lösungsansätze, da die Reproduktionskrise gar nicht als solche verstanden wird. Wie kann feministische Kritik am herrschenden Ökonomieparadigma dabei helfen, aktuelle Krisendynamiken und Ausbeutungsformen zu verstehen?

Die feministische Ökonomik (femÖk) entstammt der weltweiten Frauenbewegung Ende der 1960er Jahre, entwickelte sich seitdem in ein weites Forschungsfeld und ist bis heute mit politisch-sozialen Bewegungen verbunden. Feministische Ökonom/innen vereint das Interesse, die Rolle von Geschlechterund Herrschaftsverhältnissen zu analysieren. Ausgangspunkt ist dabei der von der Marktökonomie unbeachtete und maßgeblich unbezahlte Teil der Gesellschaft - die Reproduktionsund Sorgearbeit. Reproduktionsarbeit umfasst dabei alle Tätigkeiten, die zum Erhalt des Menschen und seines Lebensumfelds nötig sind, beispielsweise die nicht entlohnte generative Reproduktion, wie das Zeugen und Gebären von Kindern. Sorgearbeit (Care-Arbeit) ist Teil davon und meint alle haushaltsnahen Tätigkeiten wie Reinigung, das Betreuen von Kindern und Bedürftigen. Sie wird teilweise entlohnt, allerdings meist sehr schlecht. Sorgearbeit basiert auf zwischenmenschlichen Beziehungen und Kontakt. Somit folgt sie anderen Mechanismen als die der industriellen Produktion und verliert durch Rationalisierungsdruck an Qualität.

Neben der Auseinandersetzung mit dem Arbeitsbegriff untersucht die femÖK, welche Dynamiken sich hinter den gesellschaftlichen Dichotomien, wie maskulin - feminin, bezahlt unbezahlt, produktiv - reproduktiv und öffentlich - privat, verbergen. Ziel vieler feministischer Ökonom/innen ist es, ein Verständnis gesellschaftlicher Prozesse zu entwickeln, ,as if all people mattered“ (Beneria 2003; Madörin 2010; Bauhardt 2012; Ferrant et al. 2014; Exploring Economics 2016 a).

\section{Blinde Flecken des ökonomischen Mainstreams}

Gegen Ende der 1970er Jahre setzte sich die neoklassische Ökonomik als Mainstream durch und dominiert politische Analysen, (wirtschafts-)wissenschaftliche Bildung und Forschung. Auch wenn die Neoklassik unterschiedlich definiert wird, kann ein paradigmatischer Kern skizziert werden: Das Problem knapper Ressourcen. Aus der Idee, dass Individuen in reinem Selbstinteresse handeln, um ihren Nutzen zu maxi- 
mieren, folgt, dass die knappen Ressourcen optimal eingesetzt werden müssen. Somit komme es automatisch zu gesellschaftlichem Wohlstand (Exploring Economics 2016b).

Die femÖk schreibt der Neoklassik eine zweifache Geschlechterblindheit zu. Einerseits werden die Lebensrealitäten und Aktivitäten von Frauen aus ökonomischen Analysen ausgeblendet (Ferber et al. 1993). Anderseits sind Frauen bis heute kaum in der ökonomischen Wissenschaft vertreten. So liegt der aktuelle Frauenanteil unter den deutschsprachigen Professor/ innen nur bei $13 \%$ (Grimm et al. 2017). Diese Unterrepräsentation wirkt zugleich auf die Theoriebildung zurück. Daher ist (und bleibt) die Neoklassik geschlechterblind, reproduziert und verfestigt das androgyne Menschenbild unkritisch. Sie ignoriert strukturell die Machtförmigkeit der Geschlechterdimensionen. Kritisiert wird daher auch das Menschenbild der Neoklassik, der sogenannte Homo oeconomicus. Er repräsentiert ein männliches und weißes Subjekt, explizit konzipiert als nutzenmaximierend und rational agierend. Die Gleichsetzung dieser vermeintlich männlichen [1] Natur mit dem Ökonomischen suggeriert, dass es ein irrationales, weibliches und emotionales „Anderes“ gäbe. Daran schließt die Kritik an der Trennung von Markt und Haushalt an. Während die produktiv-männlichen Aktivitäten auf dem Markt stattfinden, werden die weiblichen, nicht marktvermittelten Tätigkeiten dem Privaten zugeordnet (Habermann 2008; Exploring Economics 2016a). Unbezahlte Arbeit gilt als unproduktiv und nicht wertschaffend. Dies vernachlässigt die Notwendigkeit der reproduktiven Tätigkeiten für die ökonomische Produktion und schließt sie aus Analysen und Indikatoren, beispielsweise dem Bruttoinlandsprodukt, aus (Exploring Economics 2016a).

\section{Das Paradoxon unseres Wirtschafts- systems}

Reproduktive Tätigkeiten zu vernachlässigen, führt zu einem Widerspruch in sich: Unser Wirtschaftssystem hängt von sozialreproduktiven Aktivitäten ab, klammert diese jedoch aus. Damit wird Sorgearbeit als eine unentgeltlich und unendlich verfügbare Ressource erachtet, die gesellschaftlich angeeignet und verwertet wird - das neoklassische Paradigma der Knappheit gilt nur für eingepreiste Ressourcen. Bis Ende der 1960/70er waren Frauen in Deutschland meist nicht oder nur teilzeitbeschäftigt und verrichteten allein die Sorgearbeit. Dies wandelte sich mit der Ölpreiskrise in den 1970ern. Ein „männlicher Brotverdiener" konnte die Familie nicht mehr allein ökonomisch absichern. Frauen traten massiv in den Arbeitsmarkt ein, die traditionelle Rolle der Hausfrau verlor an Bedeutung und die tägliche Sorgearbeit wurde parallel zur Erwerbszeit verrichtet. Zudem wurden soziale Sicherungssysteme aus Kostengründen abgebaut. Diese waren jedoch für die Reproduktion der Arbeitskraft nötig. Ihr Abbau verschob sorgende Aktivitäten, die nach wie vor nicht ökonomisch anerkannt wurden, zurück in die Familien. Deshalb sind staatliche und private Care-Aktivitäten bis heute schlecht bezahlt. Es folgte eine

\author{
„Die kapitalistische \\ Wachstums- und \\ Mehrwertproduktion \\ ignoriert die Produktivität \\ der Sorgearbeitenden, \\ auf der sie basiert."
}

Doppelbelastung der Frauen, verstärkt durch längere und flexiblere Arbeitszeiten, steigende Leistungsansprüche und eingeschränkte staatliche Betreuungsangebote (Fraser 2016; Winker 2013; Bauhardt 2012). Die sozialreproduktiven Aktivitäten fungieren seitdem wie ein „sozialer Air-Bag“ und müssen sich unter wachsendem Zeitdruck und Überforderung behaupten (Wichterich 2009). Das findet nicht nur zulasten der Sorgenden, sondern auch der Umsorgten statt.

Die kapitalistische Wachstums- und Mehrwertproduktion ignoriert die Produktivität der Sorgearbeitenden, auf der sie basiert, und destabilisiert sie zeitgleich massiv. Dieser Widerspruch zwischen sozialer Reproduktion und ökonomischer Produktion ist Quelle der Instabilität, der Krise der sozialen Reproduktion und somit Teil der Vielfachkrise. Die Krise der sozialen Reproduktion und einige von ihren eingangs skizzierten Symptomen sind nicht zufällig entstanden: Die Probleme wurzeln tief in unserem aktuellen Wirtschaftskonzept und gefährden langfristig die sozialen Mechanismen unserer Gesellschaft (Fraser 2016).

\section{Mit feministischer Perspektive ein Weg aus der Krise?}

Feministische Ökonomiekritik zeigt, dass lebensnotwendigen Tätigkeiten im wissenschaftlichen und politischen Diskurs kaum Beachtung geschenkt wird, und verdeutlicht die Konfliktlinien des modernen Kapitalismus. Die femÖk offenbart den systemischen Charakter der Krise. Ein „Weiter so!“ kann es also nicht geben. Doch wie kann ein „Anders weiter!“ aussehen?

Elinor Ostrom - die bis heute einzige weibliche Wirtschaftsnobelpreisträgerin - beschreibt in ihrem Commons-Ansatz eine Ökonomie des Gemeinsamen. Der Ansatz stellt die gemeinschaftliche Nutzung von geteilten Ressourcen beziehungsweise die "Organisation von Kooperation" ins Zentrum des Wirtschaftens. Zugleich zeigt Ostrom, dass die „Tragik der Allmende“ - die Übernutzung von Gemeingütern durch Eigennutz - verhindert werden kann. Mit Ressourcen sind Güter wie Atmosphäre Wasser, Wald, Fischbestände sowie soziale Beziehungen, Arbeitskraft oder Wissen gemeint. Ostroms Ansatz 
ermöglicht es also, endliche Ressourcen im Wirtschaftssystem kollektiv nachhaltig zu verwalten und zu verteilen.

Die von Riane Eisler (2014) angestoßene „Care-Revolution“-Bewegung liefert weitere Komponenten. Sie betrachtet Sorgeaktivitäten wieder als Kernaufgabe der Gesellschaft und schlägt politische Maßnahmen vor, wie eine bedürfnisorientierte Care-Ökonomie aussehen kann. Care ins Zentrum des Wirtschaftens zu rücken, bedeutet gleichzeitig unser Produktions- und Konsumverhalten neu zu denken. Denn nur so werden Arbeit(skraft) und Ressourcen wieder gewürdigt. Eisler fordert daher eine Neuordnung von Machtverhältnissen - sowohl von Geschlecht, als auch von Natur. Eine Care-Ökonomie baut somit die miteinander verwobenen Ungleichheiten ab und kann echtes nachhaltiges Wirtschaften und Zusammenleben ermöglichen.

Frigga Haugs Ansatz der sogenannten 4-in-1-Perspektive beschäftigt sich konkret mit dem Arbeitsbegriff. Sie schlägt vor, dass jeder Mensch sich durchschnittlich jeden Tag für jeweils vier Stunden der Reproduktionstätigkeit, der eigenen kulturellen Entwicklungstätigkeit, der Erwerbstätigkeit und dem gesellschaftlichen Engagement widmet - die verbleibenden acht Stunden des Tages sind für Schlaf gedacht. Obwohl auch dieses Konzept eine komplette gesellschaftspolitische Neustrukturierung erfordert, erfährt die Sorgearbeit schon auf dem Weg dahin die notwendige strukturelle Wertschätzung. Es entsteht eine nachhaltige Welt des Arbeitens und Wirtschaftens, die wieder stärker den menschlichen und natürlichen Lebensbedürfnissen dient. Um dies zu unterstützen ist in Deutschland am 8. März 2019 ein Frauenstreik geplant. Dieser ist inspiriert vom Frauenstreiktag 2018 in Spanien, wo über fünf Millionen Frauen auf die Straße gegangen sind und Erwerbs- und/oder Sorgearbeit niedergelegt haben.

\section{Dominante Denkweisen und Diskurse hinterfragen}

Auch wenn feministische Gegenkonzepte nicht allein alle Fragen der Vielfachkrise beantworten können, sind sie doch unerlässlich, um nicht nur Krisendynamiken $\mathrm{zu}$ verstehen, sondern auch sozialere, nachhaltigere und gerechtere Gesellschaftsbilder zu zeichnen - in wissenschaftlichen Diskursen, politischen Analysen und im alltäglichen Zusammenleben. Eine solche feministische Gesellschaft ist solidarisch, schützt die eigenen Lebensgrundlagen, handelt gemeinschaftlich und ermöglicht allen ein selbstbestimmtes Leben mit echter politischer Partizipation, da sie globale Ausbeutungs- und Herrschaftsverhältnisse abbaut.

Zudem müssen immer wieder dominante (wirtschafts-)wissenschaftliche und soziale Denkweisen, Strukturen und Lernprozesse aller gesellschaftlichen Akteur/innen und Institutionen hinterfragt und letztlich verändert werden. Nur so kann das herrschende Paradigma von Profit, Wettbewerb und Leistung einer progressiven feministisch-emanzipatorischen Erzählung weichen. Es ist an der Zeit!

\section{Anmerkung}

[1] Bei den im Text verwendeten Kategorien wie „Frau“ wird darauf hingewiesen, dass diese Einordnung konstruiert ist. Es sollen daher alle damit gemeint sein, die sich gemäß ihrer Geschlechtsidentität als Frau verstehen. Dazu gehören auch Personen, die von anderen als Frau wahrgenommen werden möchten, unabhängig von dem ihnen bei der Geburt zugewiesenen Geschlecht.

\section{Literatur}

Bauhardt, C. (2012): Feministische Ökonomie, Ökofeminismus und Queer Ecologies - feministische-materialistische Perspektiven auf gesellschaftliche Naturverhältnisse. Berlin, Gender Politik Online.

Bauhardt, C. (2014): Solutions to the crisis? The Green NewDeal, Degrowth, and the Solidarity Economy: Alternatives to the capitalist growth economy from an ecofeminist economics perspective. In: Ecological Economics 102: 60-68.

Benería, L. (2003): Gender, development, and globalization: economics as if all people mattered. New York, Routledge.

Demirović, A./Dück, J./Becker, F./Bader, P. (Hrsg.) (2011): Die Vielfachkrise. Hamburg, VSA: Verlag.

Exploring Economics (2016a): Feministische Ökonomik. www.exploringeconomics.org/de/orientieren/feministische-oekonomik/

Exploring Economics (2016 b): Neoklassik. www.exploring-economics.org/ de/orientieren/neoklassik/

Ferber, M. A./Nelson, J. A. (Hrsg.) (1993): Beyond Economic Man. Feminist Theory and Economics. Chicago, University of Chicago Press.

Ferrant, G./Pesando, L. M./Nowacka, K. (2014): Issues Paper: Unpaid Care Work: The missing link in the analysis of gender gaps in labour outcomes. OECD Development Centre. www.oecd.org/dev/development-gender/ Unpaid_care_work.pdf

Fraser, N. (2016): Contradictions of Capital and Care. In: New Left Review 100: 99-117.

Grimm, C./Kapeller, J./Pühringer, S. (2017): Zum Profil der deutschsprachigen Volkswirtschaftslehre. www.fgw-nrw.de/fileadmin/user_upload/FGW_ Impuls-NOED-02-Kapeller-A2-Web.pdf

Habermann, F. (2008): Der homo oeconomicus und das Andere. BadenBaden, Nomos.

Haidinger, B./Knittler, K. (2014): Feministische Ökonomie. INTRO. Wien, Mandelbaum.

Hobler, D./Klenner, C./Pfahl, S./Sopp, P./Wagner, A. (2017): Wer leistet unbezahlte Arbeit? Hausarbeit, Kindererziehung und Pflege im Geschlechtervergleich. Aktuelle Auswertungen aus dem WSI GenderDatenPortal. In: WSI Report 35. Düsseldorf, Hans-Böckler-Stiftung.

Madörin, M. (2010): Care Ökonomie - eine Herausforderung für die Wirtschaftswissenschaften. In: Bauhardt, C./Çağlar, G. (Hrsg.): Gender and Economics. Feministische Kritik der politischen Ökonomie. Wiesbaden, VS Verlag für Sozialwissenschaften. 81-104.

Winker, G. (2013): Zur Krise sozialer Reproduktion. In: Baumann, H. et al. (Hrsg.): Care statt Crash. Sorgeökonomie und die Überwindung des Kapitalismus. Zürich, Edition 8. 119-133.

Wichterich, C. (2009): Krisen, Kapital und Kosten. Ein feministischer Blick auf die globalen Krisen. www.sandimgetriebe.attac.at/8147.html

\section{AUTORIN + KONTAKT}

Lisa Weinhold ist in sozialen Bewegungen aktiv und beim netzwerk $\mathrm{n}$ als Projektkoordinatorin tätig, einem Verein, der studentisches Nachhaltigkeitsengagement an Hochschulen im Sinne der nachhaltigen Entwicklung fördert.

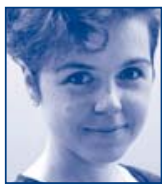

netzwerk n, c/o Thinkfarm, Moosdorfstraße 7-9, 12435 Berlin. Tel.: +49 15753054007 , E-Mail: lisa.weinhold@netzwerk-n.org 